\title{
Consultative Total Parenteral Nutrition Teams: The Effect on the Incidence of Total Parenteral Nutrition-Related Complications
}

\author{
Michael J. Dalton, Pharm.D.,* Greg Schepers, R.Ph., $†$ Joseph P. Gee, Pharm.D., $\ddagger$ \\ Cary C. Alberts, Pharm.D., § Frederick E. Eckhauser, M.D.,\| and \\ Duane M. Kirking, Pharm.D., Ph.D. I
}

From the University of North Carolina and Burroughs Wellcome Co., Research Triangle Park, North Carolina; Veterans Administration Medical Center, School of Pharmacy and School of Public Health, University of Michigan, Ann Arbor, Michigan

\begin{abstract}
A two part, prospective study was undertaken to establish the existing frequency of total parenteral nutrition(TPN) related mechanical, metabolic, and septic complications in an institution with a consultative TPN team (group A) and to determine if increasing the involvement of the TPN team in patient monitoring and verifying adherence to TPN infection control guidelines would reduce the incidence of these complications (group B). The initial 28 consecutive patients were managed entirely by their primary physicians with the role of the TPN team limited to consultative activities while the next 29 patients receiving TPN were managed jointly by their primary physicians and the TPN team. Analysis of the results show group B to have a significant reduction in metabolic
\end{abstract}

The initial enthusiasm for the use of total parenteral nutrition (TPN) therapy has been tempered somewhat by isolated reports ${ }^{1-8}$ and multicenter surveys $\mathrm{s}^{9,10}$ suggesting a high incidence of therapy-related complications. These complications can be classified as mechanical, metabolic, or septic abnormalities. Kaminski and Stolar ${ }^{9}$ reported a mechanical complication rate of $12.1 \%$ and more recently Nehme ${ }^{11}$ noted a much higher incidence of $33.5 \%$. Kaminski and Stolar ${ }^{9}$ also reported a $36.7 \%$ occurrence of electrolyte imbalances and a $27.4 \%$ incidence of significant glycosuria. They identified inadequate patient monitoring as the primary cause of these complications. With regard to catheter sepsis, infection rates as high as $42 \%$ have been reported. ${ }^{9}$ However, by adhering to strict infection control guidelines, Ryan et $\mathrm{al}^{12}$ were able to reduce the rate of catheter sepsis from 20 to $3 \%$.

Received for publication, February 17, 1983.

Accepted for publication, May 25, 1983.

Reprint requests to: Michael J. Dalton, Pharm.D., Clinical Research Fellow, Burroughs Wellcome Company, 3030 Cornwallis Road, Research Triangle Park, NC 27709.

* Clinical Research Fellow, University of North Carolina and Burroughs Wellcome Co, Research Triangle Park, NC 27709.

+ TPN Team Coordinator, Veterans Administration Medical Center, Ann Arbor, MI.

$\Varangle$ Assistant Professor of Pharmacy, School of Pharmacy, University of Michigan, Ann Arbor, MI

$\$$ Clinical Coordinator. Veterans Administration Medical Center, Ann Arbor, MI.

If Chief of Surgery. Veterans Administration Medical Center. Ann Arbor. MI.

- Assistant Professor of Pharmacy, School of Pharmacy and Assistant Professor of Medical Care Organization, School of Public Health, University of Michigan, Ann Arbor, MI. complications, decreased incidence of mechanical abnormalities, and approximately equal incidences of documented sepsis. However, when compared to the results of an institution in which the TPN team has complete control of TPN therapy, even the group $B$ patients had a relatively excessive number of TPN-related complications especially in the categories of mechanical and metabolic abnormalities. Thus, consultative TPN teams do not necessarily ensure optimum TPN therapy and institutions using this approach to provide nutrition parenterally must be prepared to establish the incidence of TPN-related complications and to expand the involvement of the TPN team as required to control the frequency of these anomalies. (Journal of Parenteral and Enteral Nutrition 8:146-152, 1984)

To minimize the occurrence of TPN-related complications, the development of multidisciplinary teams to manage TPN therapy has been advocated. ${ }^{1,10-17}$ As a result many institutions have developed TPN teams to assume primary responsibility for managing all patients receiving TPN therapy. Studies evaluating these teams have demonstrated substantial therapeutic and economic benefits. ${ }^{1,10-12,17}$ Recently, two groups of patients receiving TPN therapy were compared prospectively with one group managed solely by a nutrition team and the other by private physicians without input from the TPN team. The incidence of mechanical, metabolic, and septic complications was significantly greater in the group not managed by the nutrition team. ${ }^{11}$

Another approach to the team concept has been the development of consultative TPN teams which allow utilization of already available manpower and financial resources. Most of the literature concerning consultative teams has been descriptive reports or studies looking only at the value of these teams in reducing septic complications. ${ }^{14-16,18,19}$ Although Hickey et $\mathrm{al}^{19}$ showed an overall reduction in metabolic and infectious problems after the initiation of a consultation service, they advised caution in interpreting their results because of a small patient population. Thus, the value of consultative TPN teams in minimizing mechanical, metabolic, and septic complications has yet to be firmly established by well controlled studies with adequate patient populations.

The Ann Arbor Veterans Administration Medical Center (AAVAMC), a general medical and surgical teaching hospital affiliated with the University of Michigan, established a consultative TPN team in the fall of 1979 . Team members include a physician, several pharmacists, 
a nurse, and a dietitian. The consultative nature of the AAVAMC TPN team dictates that ultimate responsibility for all decisions relating to TPN therapy rests with the patients' primary physicians and that the usefulness of the TPN team is limited by its degree of acceptance and utilization by the primary physicians. At the AAVAMC, the primary physicians are usually interns and residents that rotate to the hospital as part of their educational experience and who vary greatly in their knowledge of TPN therapy.

Because of the lack of documentation on the effectiveness of purely consultative TPN teams in minimizing complications, this prospective study was undertaken to evaluate certain important aspects of the quality of TPN therapy at the AAVAMC. In addition, this study facilitated investigating the effect of some practical modifications of a consultative approach to managing TPN therapy on the incidence of TPN related complications. The specific objectives of this study were:

(1) To establish the existing frequency of TPN related mechanical, metabolic, and septic complications at the AAVAMC.

(2) To determine if increasing the involvement of the AAVAMC's TPN team in patient monitoring and in verifying adherence to TPN infection control guidelines would significantly increase both the level of compliance with TPN patient monitoring policies and the degree of compliance with TPN infection control guidelines over that observed with the existing system.

(3) To determine if increasing the involvement of the AAVAMC's TPN team in patient monitoring and verifying adherence to TPN infection control guidelines would significantly reduce the frequency of TPN related mechanical, metabolic, and septic abnormalities compared to the existing incidence of these complications.

\section{METHODS}

\section{Patient Selection and TPN Team Activities}

AAVAMC patients requiring TPN during a 9-month period from June 15, 1981 to March 1, 1982 were included in this prospective study. The initial 28 consecutive patients were managed according to existing procedures at the AAVAMC (group A). Thus, the primary physicians for these patients were responsible for all decisions relating to TPN therapy. After a 2 -wk between groups washout period, the next 29 consecutive patients receiving TPN therapy were managed jointly by their primary physicians and the TPN team (group B). In addition to their usual consultative activities, the TPN team also assumed responsibility for the following functions:

(1) Before the initiation of therapy, the TPN team reviewed each patient's medical record to determine the indication for parenteral nutrition and performed baseline anthropometric measurements. In situations where the patients were not considered to be candidates for TPN therapy, the primary physicians were contacted and alternative therapy recommended. The final decision whether or not to initiate TPN was left up to the primary physicians; however, in some cases the TPN team requested other individuals such as the Senior Medical
Resident, the Chief of Medicine, or the Chief of Surgery to review questionable decisions.

(2) The TPN team verified that selected observable infection control guidelines had been followed during the catheter insertion process and that central catheter placement was confirmed by a chest $x$-ray. In cases where violations of these guidelines were noted. TPN therapy was withheld until corrective action had been taken or until approval for the initiation of therapy was obtained from the Chairman of the TPN team.

(3) The TPN team assumed responsibility for ensuring that all laboratory tests and other TPN patient monitoring parameters were obtained. This was accomplished by placing a standardized physicians' order form into the patient's medical record containing nursing orders and laboratory testing procedures to be followed while the patient was on TPN therapy unless otherwise specified.

(4) The TPN team continued to verify on a daily basis that TPN infection control guidelines such as daily dressing and tubing changes, use of an infusion device, and the proper use of the TPN line were being followed. Violations of these guidelines were reported to the appropriate responsible individuals.

Other aspects of TPN therapy such as selection and daily ordering of the TPN solution continued to be the responsibility of the patient's primary physician.

\section{Data Collection-TPN-Related Complications}

Data for both groups of patients were collected by the primary investigators via daily monitoring of patients medical records and attending weekly TPN teams rounds. Only the Chairman of the TPN team and the Clinical Pharmacy Coordinator were aware of the details of this study or of the activities of the primary investigators.

Mechanical complications were defined as technical complications of subclavian vein catheterization. ${ }^{9,11}$ Any structure in the upper chest or root of the neck injured secondary to catheterization as well as other catheter related complications such as air embolism and catheter malposition were recorded.

Metabolic complications included abnormalities in electrolyte status, $\mathrm{pH}$, glucose, and vitamin $\mathrm{K}$. Electrolyte, $\mathrm{pH}$, and blood glucose abnormalities were defined as serum levels beyond the normal physiological range occurring during TPN therapy and not corrected with 24 hr. ${ }^{11}$ Complications of glucosuria were defined as urine sugar levels of $3+$ or $4+$, using a glucose oxidase method (Testape), for one or more days. ${ }^{9}$ An exception to the 24hr correction period was inorganic phosphate in which case a complication was defined as any level outside the normal physiologic range occurring after the initiation of therapy. Additionally, any incidence of an increased prothrombin time outside the normal physiologic range developing during TPN therapy and responding to vitamin $\mathrm{K}$ was classified as a complication.

A septic complication was defined as an episode of sepsis where no other anatomic locus for this infection could be identified other than the TPN catheter and which resolved upon removal of the catheter." Confir- 
mation of sepsis required a positive blood culture or an infected catheter tip.

\section{Data Collection-TPN Patient Monitoring Parameters}

The level of compliance with TPN patient monitoring policies was determined by measuring the frequency with which laboratory tests and other patient monitoring parameters were ordered and obtained and then comparing the results to the monitoring standards for the three stages of TPN therapy.,18,20,21 The baseline stage was defined as the 7-day period immediately prior to the initiation of therapy. The stabilization stage refers to the first 7 days of therapy. The third stage of therapy, the maintenance stage, was the period of TPN therapy after the stabilization interval.

\section{Data Collection-Degree of Compliance with Established Infection Control Guidelines}

The degree of compliance with established infection control policies for each group was determined by monitoring for violations of the more observable of these procedures. Included were all incidences of noncompliance with the following guidelines:

(1) Central venous catheter insertion should be effected away from the patient care area (use treatment room).

(2) Sterile technique should be used during central venous catheter insertion (gown, cap, gloves, and mask to be worn).

(3) Proper positioning of the central venous catheter after insertion should be established with a chest $\mathrm{x}$-ray prior to initiating therapy.

(4) A continuous infusion pump should be used to control the administration rate of TPN fluid (patients receiving peripheral TPN should have infusion pumps controlling both the amino acid-glucose mixture and the fat emulsion).

(5) In patients receiving peripheral TPN, the amino acid-glucose mixture and the fat emulsion should be administered simultaneously.

(6) TPN dressings should be changed every $24 \mathrm{hr}$.

(7) TPN tubing should be changed at least every 24 hr.

(8) The line through which the TPN fluid is being administered should not be used for other purposes such as measuring central venous pressure, administering blood products, or the infusion of other medications.

\section{Statistical Analysis}

Student's $t$-test was used to evaluate differences in age, pretreatment percentage of ideal body weight, duration of therapy, number of metabolic complications per patient, number of metabolic complications per patient among those patients having a complication, number of metabolic complications per patient per treatment day, and the number of metabolic complications per patient per treatment day among those patients having a complication. An appropriate $t$-test was used to correct for unequal variances when they occurred. The $\chi^{2}$ test was used to evaluate differences in the percent of patients treated by medical versus surgical physicians, peripheral versus central TPN, treatment location within the hospital, level of compliance with patient monitoring guidelines, the number of central catheters pulled without documentation of sepsis, and the number of patients in which a metabolic complication was observed. The difference in the number of deaths during TPN therapy was evaluated using Fisher's exact test.

\section{RESULTS}

The incidence of TPN-related complications, the level of compliance with TPN patient monitoring policies, and the degree of adherence to TPN infection control guidelines were determined for each group. Improvement was demonstrated in each of these categories for the group B patients although this difference was not always statistically significant.

\section{Treatment Groups}

Upon initiating this study, the first 28 patients treated with TPN therapy were designated group A. Twentynine of the next 32 patients referred for TPN therapy were designated group B. Three patients were not treated with TPN therapy because of team recommendations to use an alternative method to provide nutrition. In addition, TPN therapy for three other group B patients was discontinued early because of TPN team recommendations. These patients either no longer required parenteral nutrition to maintain their nutritional status or were considered terminal. No significant differences were found between the groups except in the percent days central versus peripheral TPN (Table I) and each group had a similar distribution of diagnoses (Table II).

\section{Mechanical Complications}

The number of catheters placed and their resulting mechanical complications are shown in Table III. The most serious problems were the cases of air embolism occurring in three group A patients when the administration tubing inadvertently disconnected from the central catheter. One of these patients required transportation to another hospital in order to be placed in a hyperbaric chamber. All other patients recovered without further complications. Overall, $35 \%$ of the patients in group A experienced a mechanical complication compared to $11 \%$ in group $B$. This difference was marginally significant $(p<0.1)$.

\section{Metabolic Complications}

A detailed comparison of the observed occurrence of metabolic imbalances is included in Table IV. The treatment groups were examined for differences in the percentage of patients who had an abnormality, number of episodes per patient, number of episodes per patient among those patients having a complication, the number of episodes per patient per treatment day, and the number of episodes per patient per treatment day among those patients having a complication. The imbalance rate was less for patients in group $B$ in all situations except 
TABLE I

Patient population characteristics ${ }^{a}$

\begin{tabular}{lll}
\hline \multicolumn{1}{c}{ Parameter } & \multicolumn{1}{c}{ Group A } & Group B \\
\hline Age & $60.0 \pm 15.6$ & $59.9 \pm 10.9$ \\
Sex (no. of males) & 27 & 28 \\
Pretreatment \% IBW $\dagger$ & $0.90 \pm 0.18$ & $.94 \pm .17$ \\
Duration of TPN (days) & $12.4 \pm 9.2$ & $10.6 \pm 7.1$ \\
\% Days medicine service & 35.6 & 32.6 \\
\% Days surgery service & 64.4 & 67.4 \\
\% Days central TPN & 63.2 & 74.6 \\
\% Days peripheral TPN & 36.8 & 25.4 \\
\% Days MICU & 24.7 & 31.6 \\
\% Days 4 west & 38.5 & 36.8 \\
\% Days other & 36.8 & 31.6 \\
Deaths during TPN & 2 & 6
\end{tabular}

${ }^{a} \chi^{2}$ and $t$-test, no significant difference except \% days central TPN $(p<0.005)$.

+ Ideal body weight.

TABle II

Underlying diseases

\begin{tabular}{lcc}
\hline \multicolumn{1}{c}{ Condition } & Group A & Group B \\
\hline Cancer & 10 & 10 \\
Head/neck/lung & 4 & 3 \\
Gastrointestinal & 5 & 6 \\
Bladder & 1 & 0 \\
Leukemia & 0 & 1 \\
Pancreatitis & 2 & 1 \\
Obstruction & 9 & 10 \\
Crohns/ulcerative colitis & 2 & 3 \\
Acute renal failure & 1 & 1 \\
Abdominal aortic aneurysm & 1 & 1 \\
Intra abdominal abscess & 0 & 1 \\
Aortic-femoral bypass & 2 & 1 \\
Gastrointestinal bleeding & 1 & 1 \\
Total & 28 & 29 \\
\hline
\end{tabular}

TABLE III

Mechanical complications

\begin{tabular}{lcc}
\hline \multicolumn{1}{c}{ Condition } & Group A & Group B \\
\hline No. of catheters placed & 25 & 20 \\
No. of patients & 20 & \multicolumn{2}{c}{18} \\
Complications & $7(35 \%)$ & $2(11 \%)$ \\
Air embolism & $3(15 \%)$ & 0 \\
Catheter malposition & $2(10 \%)$ & 0 \\
Catheter falling out (jugular) & $1(5 \%)$ & $1(5.5 \%)$ \\
Disconnections & $1(5 \%)$ & 0 \\
Subclavian artery puncture & 0 & $1(5.5 \%)$ \\
\hline
\end{tabular}

the number of bicarbonate abnormalities per patient per treatment day among those patients having a bicarbonate abnormality, and the number of prothrombin time imbalances per patient among those patients having a prothrombin time abnormality. None of the situations when the complication rate was greater for group B was statistically significant whereas the decreases in complications observed for group B patients were frequently statistically significant. Evidence for an overall trend toward more favorable results in group B patients is suggested by the highly significant differences in the mean number of episodes per patient $(p<0.0005)$, the mean number of episodes per patient among those patients having a complication $(p<0.025)$, and the total number of episodes per patient per treatment day $(p<$ 0.005) (Table IV). Magnesium was not included in the data analysis because less than $50 \%$ of the patients in group A had any levels drawn.

\section{Septic Complications}

Both treatment groups had one episode of documented catheter sepsis (Table V). The group B patient subsequently developed systemic candidiasis requiring longterm treatment with Amphotericin B before clearing. Also recorded for each group was the number of catheters that were pulled because of suspected sepsis but without subsequent documentation of sepsis. This occurred on nine occasions in group A $v s$ two in group $\mathrm{B}(p<0.05)$.

\section{Patient Monitoring}

The level of compliance with TPN patients monitoring policies as contained in the AAVAMC TPN team protocol was determined for each group (Table VI). These tests were classified as being either a nutritional, metabolic, or miscellaneous patient monitoring parameter, as well as being divided into baseline, stabilization, and maintenance monitoring periods. It should be noted that no attempt was made to weight the tests by the frequencies at which they were supposed to be obtained. During both the stabilization and maintenance periods, the patients in group $B$ had highly significant increases in the level of patient monitoring compared to group A $(p<$ 0.0005 ). Although Group B patient monitoring was also improved during the baseline period, the difference was not statistically significant.

\section{Adherence to Infection Control Guidelines}

Table VII shows the degree of adherence to infection control guidelines. Overall, the results were very similar for each group except in utilizing sterile technique during the catheter insertion procedure. Failure to wear caps, gowns, or mask when inserting the catheter was the most common violation. The low percentage of patients who had catheters inserted away from the treatment area, $20 \%$ for group A compared to $25 \%$ in group B, reflects a hospital decision not to enforce this policy. Other than using an infusion pump with fat emulsion during peripheral TPN, adherence to other infection control guidelines was greater than $90 \%$ for each group and, therefore, statistical analysis of the differences between the groups was not performed.

\section{DISCUSSION}

\section{First Objective}

The results from group A reflect the existing level of TPN related complications in an institution with a consultative TPN team. Thus, an evaluation of this data will provide insight into the value of this type of team in minimizing these abnormalities. However, it should be noted that because reports describing complication rates during TPN therapy rarely use exactly the same criteria when defining an abnormality, discrepancies in complication rates do not necessarily indicate real differences in results.

The $35^{c} c$ incidence of mechanical complications in 
TABLE IV

Metabolic complications

\begin{tabular}{|c|c|c|c|c|c|c|c|c|c|c|}
\hline \multirow{2}{*}{ ABN } & \multicolumn{2}{|c|}{ I } & \multicolumn{2}{|c|}{ II } & \multicolumn{2}{|c|}{ III } & \multicolumn{2}{|c|}{ IV } & \multicolumn{2}{|c|}{$\mathrm{V}$} \\
\hline & A & B & $A$ & B & A & $\mathrm{B}$ & $\mathrm{A}$ & B & A & B \\
\hline $\mathrm{Na}$ & 57.1 & 41.4 & 3.9 & $1.7^{b}$ & 6.8 & $3.7^{b}$ & 0.31 & 0.16 & 0.55 & 0.36 \\
\hline $\mathbf{K}$ & 35.7 & 24.7 & 1.1 & 0.5 & 3.2 & 2.0 & 0.09 & 0.05 & 0.26 & 0.19 \\
\hline Cl & 75 & $34.5^{c}$ & 3.7 & $1.0^{c}$ & 4.8 & 2.9 & 0.28 & $0.08^{c}$ & 0.38 & 0.23 \\
\hline $\mathrm{CO}_{2}$ & 75 & $44.8^{b}$ & 3.4 & 2.0 & 4.8 & 4.5 & 0.29 & 0.17 & 0.38 & 0.38 \\
\hline $\mathrm{Ca}$ & 21.3 & $0^{b}$ & 2.7 & $0^{b}$ & 2.7 & $0^{c}$ & 0.08 & 0 & 0.36 & $0^{d}$ \\
\hline $\mathbf{P}$ & 57.1 & 42.9 & 2.5 & 1.5 & 4.3 & 3.5 & 0.24 & 0.26 & 0.43 & 0.38 \\
\hline $\mathbf{B G}$ & 46.4 & 34.5 & 2.5 & 1.5 & 5.4 & 4.3 & 0.23 & 0.13 & 0.50 & 0.37 \\
\hline UG & 10.7 & 0 & 0.3 & 0 & 2.3 & $0^{d}$ & 0.02 & 0 & 0.18 & $0^{d}$ \\
\hline PT & 52.2 & $13.8^{d}$ & 1.5 & 0.5 & 2.8 & 3.8 & 0.15 & 0.04 & 0.30 & 0.29 \\
\hline $\mathbf{T}$ & 47.8 & $26.3^{d}$ & 2.2 & $1.1^{c}$ & 4.6 & $3.6^{b}$ & 0.19 & $0.11^{d}$ & 0.38 & 0.33 \\
\hline
\end{tabular}

${ }^{a}$ ABN, abnormality; I, \% of patients with complication; II, no. of abnormal episodes per patient; III, no. of episodes per patient among those patients having an abnormality; IV, no. of abnormal episodes per patient per treatment day; $V$, no. of episodes per patient per treatment day among those patients having an abnormality; BG, blood glucose; UG, urine glucose; PT, prothrombin time; T, mean no. of complication in that category.

${ }^{b} p<0.05$.

${ }^{c} p<0.005$.

${ }^{d} p<0.0005$.

TABLE V

Catheter sepsis

\begin{tabular}{lcc}
\hline & Group A & Group B \\
\hline $\begin{array}{l}\text { Incidence } \\
\begin{array}{l}\text { Organism } \\
\text { Catheters pulled without } \\
\text { documentation of sepsis }\end{array}\end{array}$ & $\begin{array}{c}1 \\
\text { Candida/Enterobacter }\end{array}$ & Candida \\
\hline
\end{tabular}

TABLE VI

Percentage compliance with monitoring standards ${ }^{a}$

\begin{tabular}{lcc}
\hline $\begin{array}{c}\text { Period } \\
\text { classification }\end{array}$ & Group A & Group B \\
\hline $\begin{array}{lc}\text { Baseline } \\
\text { Nutritional }\end{array}$ & $\%$ & $\%$ \\
Metabolic & 32.4 & 42.7 \\
Miscellaneous & 59.8 & 56.0 \\
Stabilization $_{\text {Nutritional }}^{b}$ & 50.0 & 59.7 \\
Metabolic $^{b}$ & 54.4 & 84.7 \\
Miscellaneous $^{b}$ & 62.4 & 79.9 \\
Maintenance $^{\text {Nutritional }}{ }^{b}$ & 81.3 & 88.3 \\
Metabolic $^{b}$ & & \\
Miscellaneous $^{b}$ & 59.1 & 86.5 \\
\hline
\end{tabular}

a Nutritional tests include weight, complete blood count, $\mathrm{Fe} /$ total iron binding capacity, UUN, and creatinine. Metabolic tests include electrolytes, $\mathrm{SMA}_{12}, \mathrm{Mg}++$, and PT/PTT. Miscellaneous tests include vital signs and urine glucose. SMA A $_{12}$ contains total protein, albumin, $\mathrm{Ca}++$, phosphate, blood urea nitrogen, creatinine, bilirubin, uric acid, cholesterol, glucose, SGOT, and alkaline phosphatase.

${ }^{b} p>0.0005$.

TABLE VII

Adherence to infection control guidelines

\begin{tabular}{lcc}
\hline \multicolumn{1}{c}{ Guideline } & Group A & Group B \\
\hline & $\%$ & $\%$ \\
Catheter insertion away from patient & 20 & 22.7 \\
$\quad$ care area & 65 & 96.7 \\
$\begin{array}{l}\text { Sterile technique } \\
\text { Chest x-ray }\end{array}$ & 100 & 100 \\
Infusion pump for amino acid solu- & $\mathbf{9 2 . 8}$ & 99.7 \\
$\quad$ tion & $\mathbf{6 3 . 9}$ & $\mathbf{6 5 . 9}$ \\
$\begin{array}{l}\text { Infusion pump for fat emulsion } \\
\text { (peripheral TPN) }\end{array}$ & 94.5 & 100 \\
$\begin{array}{l}\text { Daily dressing changes } \\
\text { Daily tubing changes }\end{array}$ & $\mathbf{9 6}$ & 100 \\
Proper use of line & $\mathbf{9 2 . 8}$ & 100 \\
\hline
\end{tabular}

group A compares poorly with results reported by most institutions. ${ }^{8,9,11,19}$ Nehme $^{11}$ reported mechanical complication rates of 3 and $33 \%$ in treatment groups managed with and without TPN team control, respectively. Complication rates of less than $3 \%$ are currently being published with increasing frequency when adequate knowledge and control of the proper techniques for insertion and care of central venous catheters are present. ${ }^{8}$ Most of the abnormalities in the present study occurred as a direct result of tubing disconnections at either the catheter hub or a junction in the administration set. This suggests inadequate catheter/tubing care after the insertion process as does the dislodging of the jugular catheter. As a result of TPN team intervention, Luer loc tubing is now being used in all central catheters as well as continuing to tape all tubing junction sites. Both of the malpositioned catheters were noted on chest $x$-ray before initiating therapy and were repositioned without further difficulty.

The incidence of metabolic complications for group A also does not compare favorably with studies from other institutions with or without TPN teams..$^{8,9,11,19}$ An average of $58.3 \%$ of the patients had at least one abnormality in each of the categories evaluated. Plus, each patient had an average of 2.2 comlications for each metabolic category while on TPN therapy. Kaminski and Stolar ${ }^{9}$ reported that with minimal TPN team involvement, $36.7 \%$ of the patients had electrolyte imbalances while in Nehme's study, ${ }^{11} 54.8 \%$ of the patients controlled by private physicians had metabolic complications compared to $5 \%$ of those managed by the TPN team. Nehme allowed a 48-hr correction period for electrolytes compared to a 24-hr limit in the present study whereas in the report by Kaminski and Stolar, the correction time was either the same or shorter than the present study. Further examination of the metabolic complication results reveals fairly universally inadequate anticipation of requirements and failure to correct abnormalities rapidly once they occurred both of which are probably related to a low level of patient monitoring and less than optimal understanding of electrolyte requirements in TPN patients. A purely consultative TPN team does not appear 
to necessarily allow good control of metabolic complications.

The 5\% infection rate in group A attributable to catheter sepsis, as defined by Ryan et $a{ }^{12},{ }^{12}$ is within the range reported by investigators when adhering to good sterile technique during catheter insertion and subsequent care. $^{2,6,12}$ There was, however, a seemingly large number of catheters pulled because of suspected sepsis but without subsequent documentation. Several of these patients improved without identifying a source of infection.

\section{Second Objective}

As evidenced by Table VI, the increased involvement of the TPN team significantly improved the level of patient monitoring. Even more improvements would have been found except that laboratory tests ordered on weekends were frequently not done. The relatively poor level of patient monitoring during the baseline period was due to a hesitancy by physicians to withhold TPN therapy until baseline data could be obtained. However, given the high level of metabolic complications in both groups, early documentation of electrolytes and other monitoring parameters may be necessary to reduce these to a more acceptable frequency.

The degree of adherence to infection control guidelines for both groups was very similar. The biggest change was the improvement in sterile technique during catheter placement observed in group B. The primary physicians eventually noticed both the investigators and the TPN team were monitoring the conditions during catheter placement and this awareness probably contributed to the increased compliance with sterile technique guidelines. Neither of the groups routinely used infusion devices to administer peripheral fat emulsion reportedly because of an equipment shortage. Although patients were not specifically monitored for the incidence of phlebitis during peripheral TPN, the rate of occurrence of this abnormality seemed to be fairly high and may have been related to fat emulsion administration techniques.

\section{Third Objective}

The third objective was to determine if an increased involvement of the TPN team would decrease TPN complications. Analysis of the results show a significant reduction in metabolic complications, decreased incidence of mechanical abnormalities, and approximately equal incidences of documented sepsis but reduced numbers of suspected sepsis without documentation.

Although patients in group B tended to have fewer metabolic imbalances in all categories evaluated, the reduction was most significant for the percentage of patients with a complication, the number of episodes per patient, the number of episodes per patient among those patients with a complication, and the number of episodes per patient per treatment day. In order to determine how quickly an abnormality was corrected once it occurred, patients were evaluated in categories III and V (Table IV). The patients in group $B$ generally did better in both areas but the differences were usually not statistically significant. Despite the improvement seen in group B, these patients still had an average abnormality rate for each category of $26.3^{\circ}$. Perhaps more involvement by the TPN team in other aspects of therapy such as standardization of TPN solutions or writing TPN solution orders would help improve metabolic control.

The relatively large number of mechanical problems in group A, especially air embolisms, was probably coincidental since there is no evidence suggesting that air embolism had been a problem in the past at the AAVAMC. The increased daily monitoring by the TPN team and the equipment change to Luer loc connectors helped prevent problems in group B patients and should minimize reoccurrence in the future. Probably the best method for a TPN team to have an impact on mechanical complications other than those related to catheter care would be to assume responsibility for catheter placement. The number of problems associated with catheter placement has been shown to be related to the experience of the individual performing the procedure (8).

Changes in the TPN team had no definite effect on the incidence of catheter sepsis. However, the low level of compliance with all the recommendations for sterile technique observed in group A patients along with the number of catheters pulled because of suspected catheter sepsis causes some concern. Catheters were either being changed needlessly or possibly undocumented catheter sepsis was present. Mechanisms to ensure compliance with all sterile technique recommendations should be considered.

\section{CONCLUSION}

The existing frequency of TPN related complications at the AAVAMC (group A), reflecting the influence of a purely consultative TPN team, is greater than the reduced level of these abnormalities possible when TPN teams have more control of therapy. Modification of the TPN team (group B) resulted in increased patient monitoring and adherence to infection control guidelines as well as decreased mechanical and metabolic complications. However, the incidence of these abnormalities remained relatively excessive. Therefore, additional responsibilities and activities for the TPN team should be considered in order to further reduce TPN related complications and improve therapy.

\section{ACKNOWLEDGMENTS}

The authors acknowledge Nabil Khalidi, Pharm.D., coordinator of the University of Michigan Hospital Parenteral-Enteral Nutrition Team, for his valuable input concerning the design and conduct of this study. We also thank Baxter-Travenol Company for their support via a grant to the Ann Arbor VA Hospital to help develop a TPN team.

\section{REFERENCES}

1. Thomas RJ: The organization, methods, and experience of a parenteral nutrition service. Aust NZ J Surg 48:539-544, 1978

2. Goldman DA, Maki DG: Infection control in total parenteral nutrition. JAMA 223:1360-1634, 1973 
3. Maki DG, Goldmann DA, Rhame FS: Infection control in intravenous therapy. Ann Intern Med 79:857-887, 1973

4. Freeman JB, Lemire A, MacLean LD: Intravenous alimentation and septicemia. Surg Gynecol Obstet 135:708-712, 1972

5. Copeland EM III, MacFayden BV Jr, McGown C, et al: The use of hyperalimentation in patients with potential sepsis. Surg Gynecol Obstet 138:377-380, 1974

6. Bently DE, Lepper $M H$ : Septicemia related to indwelling venous catheters. JAMA 206:1749-1752, 1968

7. Dudrick SJ, MacFayden BV Jr, Van Buren CT, et al: Parenteral hyperalimentation problems and solutions. Ann Surg 176:259-264, 1972

8. Vazquez RM: Modern clinical nutrition-part 6: subclavian catherization. Am J Intravenous Ther Clin Nutr 7:11-29, 1980

9. Kaminski MV, Stolar MH: Parenteral hyperalimentation-a quality of care survey and review. Am J Hosp Pharm 31:228-235, 1974

10. Infection control in hyperalimentation therapy, Hospital Infections and Microbiological Control Branches, Bacterial Disease Division, Bureaus of Epidemiology. Center for Disease Control: Atlanta, GA, 1972

11. Nehme AE: Nutritional support of the hospitalized patient: the team concept. JAMA 243:1906-1908, 1980

12. Ryan JA, Abel RM, Abbott WM, et al: Catheter complications in total parenteral nutrition. N Engl J Med 390:757-760, 1980
13. Blackburn GL, Bristrian BR, Maini BS, et al: Nutritional and metabolic assessment of the hospitalized patient. JPEN 1:11-22, 1977

14. Skoutakis VA, Martinez DR, Miller WA, et al: Team approach to total parenteral nutrition. Am J Hosp Pharm 32:693-697, 1975

15. Mutchie KD, Smith KA, MacKay MW, et al: Pharmacist monitoring of parenteral nutrition: clinical and cost effectiveness. Am J Hosp Pharm 36:785-787, 1979

16. Powell-Tuck J, Farwell JA, Nielsen T: Team approach to longterm intravenous feeding in patients with gastrointestinal disorders. Lancet 2:825-828, 1978

17. Greenlaw CW: Pharmacist as team leader for total parenteral nutrition therapy. Am J Hosp Pharm 36:648-650, 1979

18. Swenson JP, Edwards D, Chamberlain M, et al: A total parenteral nutrition protocol. Drug Intell Clin Pharm 1:714-720, 1977

19. Hickey MM, Munger TO, Salen RB, et al: Parenteral nutrition utilization: evaluation of an educational protocol and consult service. JPEN 3:433-437, 1979

20. Koda-Kimble MA, Katcher BS, Young LY, et al (eds.): Applied therapeutics for clinical pharmacists, 2nd ed. Applied Therapeutics Inc, San Francisco, 1978, pp 57-70

21. Eriksson B, Douglass HO: Intravenous hyperalimentation: an adjunct to treatment of malignent disease of upper gastrointestinal tract. JAMA 243:2049-2052, 1980 